\title{
Validation of dynamic three-dimensional whole heart magnetic resonance myocardial perfusion imaging at 3.0 Tesla against fractional flow reserve for the detection of flow-limiting coronary heart disease
}

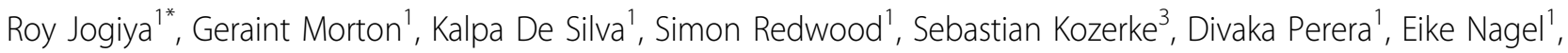 \\ Sven Plein ${ }^{2}$
}

From 15th Annual SCMR Scientific Sessions

Orlando, FL, USA. 2-5 February 2012

\section{Summary}

We demonstrate the feasibility of 3D myocardial perfusion CMR at 3 Tesla against fractional flow reserve (FFR) in 53 patients for the detection of flow-limiting coronary artery disease and show good agreement between the techniques. This technique shows excellent diagnostic sensitivity and specificity and may offer an alternative method of detecting ischaemia for the purpose of guiding revascularisation and risk stratification.

\section{Background}

Three-dimensional (3D) myocardial perfusion cardiovascular magnetic resonance (CMR) has recently been proposed to overcome the limited spatial coverage of conventional perfusion CMR methods1. The method has shown good diagnostic accuracy for the detection of coronary artery disease determined by quantitative coronary angiography (QCA)2. However the relationship between the severity of a coronary stenosis on QCA and its functional significance is variable. Pressure wirederived fractional flow reserve (FFR) $<0.75$ correlates closely with objective evidence of reversible ischemia and it has been demonstrated that ischaemia-guided PCI confers a prognostic benefit.

${ }^{1}$ Kings College London, London, UK

Full list of author information is available at the end of the article

\section{Aim}

To determine the diagnostic accuracy of whole heart 3D myocardial perfusion CMR against invasively determined FFR.

\section{Methods}

Fifty-five patients referred for angiography underwent rest and adenosine stress 3D myocardial perfusion CMR at 3Tesla (3D turbo gradient echo, flip angle 15, TR $2.0 \mathrm{~ms} / \mathrm{TE} 1.0 \mathrm{~ms}, 12$ slices of $5 \mathrm{~mm}$ thickness, in-plane resolution $2.3 \times 2.3 \mathrm{~mm} 2,10$ fold $\mathrm{k}$-space and time $\mathrm{k}-\mathrm{t}$ broad linear speed up technique acceleration with $\mathrm{k}-\mathrm{t}$ principal component analysis). Perfusion was scored visually on a patient and coronary territory basis with a score from 0-3. Ischaemic burden was calculated by quantitative segmentation of the volume of hypoenhancement. The FFR was measured in vessels with $>50 \%$ severity stenosis. Fractional flow reserve $<0.75$ was considered hemodynamically significant.

\section{Results}

Two patients were excluded (one claustrophobia, on poor image quality). From the remaining fifty-three patients and 159 coronary vessels, 64 underwent pressure wire assessment and 39 had an FFR $<0.75$ (Figure1). Sensitivity, specificity and diagnostic accuracy of CMR analysis per patient was $90 \%, 91 \%$ and $91 \%$, respectively for the detection of significant coronary artery disease. 


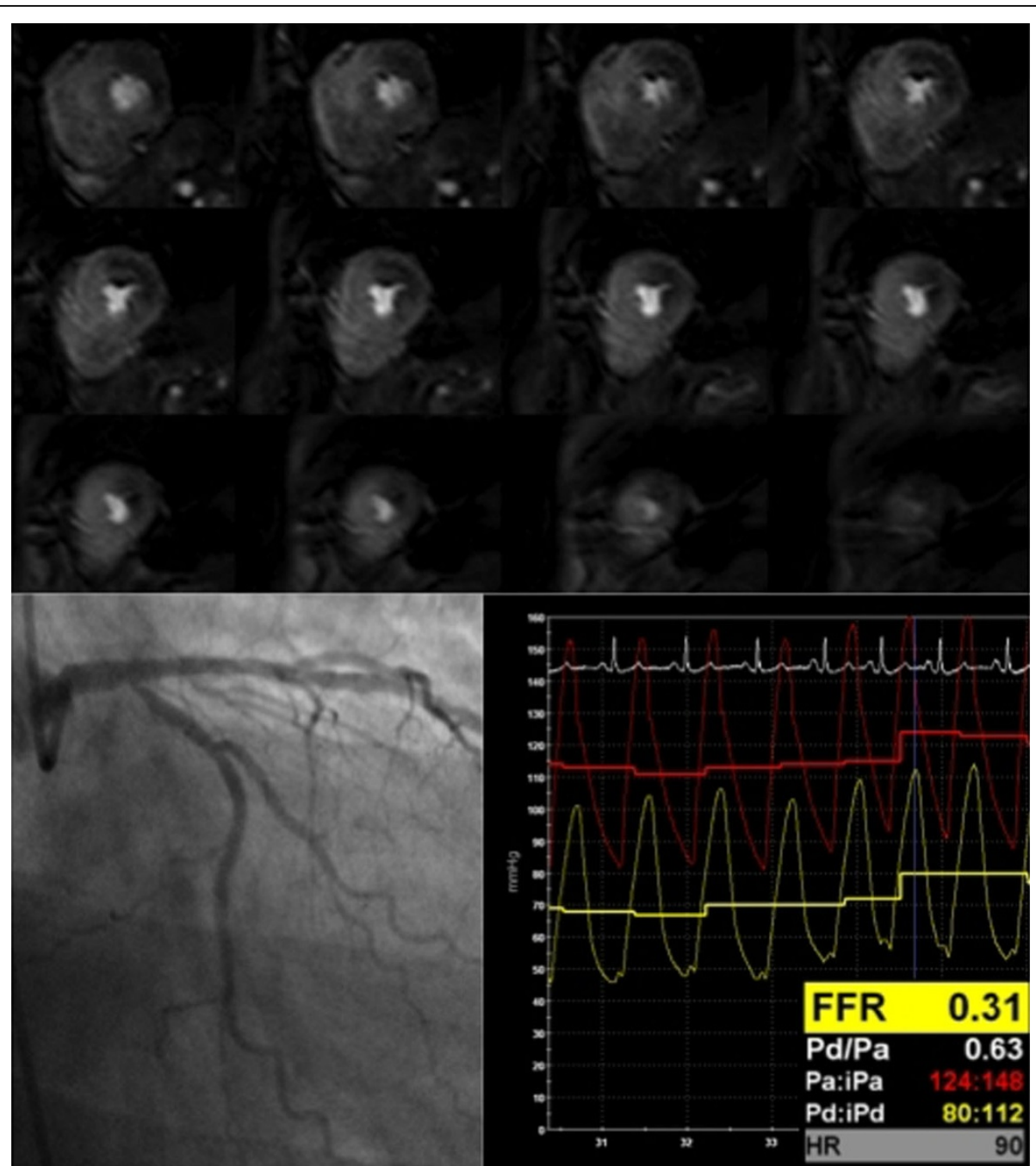

Figure $13 D$ CMR perfusion of a patient with a tight ostial circumflex lesion with positive fractional flow reserve (FFR=0.31). Note the transmural perfusion defect from the base towards the apex.

By coronary territory the values were $79 \%, 92 \%$ and $88 \%$.

\section{Conclusions}

3D CMR stress perfusion can detect functionally significant coronary artery disease with excellent sensitivity, specificity and predictive values when compared with FFR.

3D CMR perfusion imaging may offer an alternative method of detecting ischaemia for the purpose of guiding revascularisation and risk stratification.

\section{Funding}

SP is funded by British Heart Foundation fellowship FS/ 10/62/28409.

SP/EN receives research grant support from Philips Healthcare.

\section{Author details}

${ }^{1}$ Kings College London, London, UK. ${ }^{2}$ LiGHT Institute, Leeds, UK. ${ }^{3}$ ETH, Zurich, Switzerland.

Published: 1 February 2012

\section{References}

1. Vitanis V, Manka R, Giese D, Pedersen H, Plein S, Boesiger P, Kozerke S: High resolution three-dimensional cardiac perfusion imaging using compartment-based k-t principal component analysis. Magn Reson Med 2011, 65(2):575-87, doi: 10.1002/mrm.22620. Epub 2010 Oct 6.

2. Manka R, Jahnke C, Kozerke S, Vitanis V, Crelier G, Gebker R,

Schnackenburg B, Boesiger P, Fleck E, Paetsch I: Dynamic 3-dimensional stress cardiac magnetic resonance perfusion imaging detection of coronary artery disease and volumetry of myocardial hypoenhancement before and after coronary stenting. J Am Coll Cardiol 2011, 57(4):437-44.

doi:10.1186/1532-429X-14-S1-092

Cite this article as: Jogiya et al: Validation of dynamic three-

dimensional whole heart magnetic resonance myocardial perfusion imaging at 3.0 Tesla against fractional flow reserve for the detection of flow-limiting coronary heart disease. Journal of Cardiovascular Magnetic

Resonance 2012 14(Suppl 1):O92. 